OPEN ACCESS

Edited by:

Luc Pieters,

University of Antwerp, Belgium

Reviewed by:

Filomena Nazzaro,

Italian National Research Council

(CNR), Italy

Eugenia Bezirtzoglou,

Democritus University of Thrace,

Greece

Farhat Ullah,

University of Malakand, Pakistan

Javad Sharifi-Rad,

Shahid Beheshti University of Medical

Sciences, Iran

${ }^{*}$ Correspondence:

Cassandra L. Quave

cquave@emory.edu

Specialty section:

This article was submitted to

Ethnopharmacology,

a section of the journal

Frontiers in Pharmacology

Received: 02 October 2018

Accepted: 18 January 2019

Published: 06 February 2019

Citation:

Richwagen N, Lyles JT, Dale BLF and Quave CL (2019) Antibacterial

Activity of Kalanchoe mortagei and K. fedtschenkoi Against ESKAPE Pathogens. Front. Pharmacol. 10:67.

doi: 10.3389/fphar.2019.00067

\section{Antibacterial Activity of Kalanchoe mortagei and $K$. fedtschenkoi Against ESKAPE Pathogens}

\author{
Nicholas Richwagen', James T. Lyles ${ }^{1}$, Brandon L. F. Dale ${ }^{2}$ and \\ Cassandra L. Quave ${ }^{1,2,3,4 *}$ \\ 'Center for the Study of Human Health, Emory College of Arts and Sciences, Atlanta, GA, United States, ${ }^{2}$ Department \\ of Dermatology, Emory University School of Medicine, Atlanta, GA, United States, ${ }^{3}$ Emory University Herbarium, Atlanta, GA, \\ United States, ${ }^{4}$ Antibiotic Resistance Center, Emory University, Atlanta, GA, United States
}

Plants in the genus Kalanchoe (Family: Crassulaceae) are used in traditional medicine throughout the tropics for treating a variety of conditions. Two species, Kalanchoe mortagei and $K$. fedtschenkoi, have established ethnobotanical usage but have been neglected in previous research concerning their potential bioactivity. Here, we provide a thorough review of the reported antimicrobial activities of Kalanchoe genus and evaluate the in vitro antibacterial effects of two previously unexplored species against a panel of multidrug-resistant bacteria, the ESKAPE pathogens (Enterococcus faecium, Staphylococcus aureus, Klebsiella pneumoniae, Acinetobacter baumannii, Pseudomonas aeruginosa, and Enterobacter cloacae). Plant specimens were collected and voucher specimens deposited in the Emory University Herbarium. Dried plant material was ground into a powder and extracted as ethanolic macerations or as aqueous decoctions. Extracts were tested against the ESKAPE pathogens for growth inhibitory activity. Cytotoxicity to human cells was assessed via a lactate dehydrogenase assay of treated human keratinocytes (HaCaTs). K. fedtschenkoi extracts demonstrated growth inhibitory effects against two Gram-negative species, A. baumannii (strain CDC-33) and $P$. aeruginosa (AH-71), as well as $S$. aureus (UAMS-1). In these cases, growth inhibition greater than $50 \%\left(\mathrm{IC}_{50}\right)$ was generally observed at concentrations of $256 \mu \mathrm{g} \mathrm{mL}^{-1}$, though one $K$. fedtschenkoi extract (1465, prepared from stems) exhibited an $\mathrm{IC}_{50}$ against $A$. baumannii at $128 \mu \mathrm{g} \mathrm{mL} \mathrm{L}^{-1}$. All extracts were well tolerated by HaCaTs $\left(L_{50} \geq 256 \mu \mathrm{g} \mathrm{mL}^{-1}\right)$. Chemical characterization using HPLC and chemical standards established the presence of caffeic acid and quercetin in both plant species, as well as kaempferol in $K$. fedtschenkoi. These results reveal $K$. fedtschenkoi to be a plant of medicinal interest, and future research should aim to characterize the bioactivity of this species and its active constituents through bioassay-guide fractionation. Effects on bacterial biofilm formation and quorum-sensing are also research topics of interest for this genus.

Keywords: medicinal plants, MIC, phytochemicals, Crassulaceae, antibiotic resistance 


\section{INTRODUCTION}

\section{Ethnopharmacological Relevance of Kalanchoe Species}

Plants in the genus Kalanchoe (Crassulaceae), though originating mostly in Madagascar and Southeast Africa, have a global distribution in warm climates. Frequently, Kalanchoe spp. occur as exotic or invasive species. Many members of the genus are able to self-propagate from plantlets produced on the leaf margin, making established populations hard to eradicate (Descoings, 2003; Akulova-Barlow, 2009). The presence of toxic cardiac glycosides make some Kalanchoe spp. a grazing hazard for animals in agriculture, with documented issues in Brazil, South Africa, and Australia (Botha C., 2013; Botha C.J., 2013; Mendonça et al., 2018). Nevertheless, these plants display a diverse array of stunning forms and are often grown as ornamentals for their strange beauty.

Despite their often exotic presence, Kalanchoe spp. have ethnobotanical uses wherever they are found, sometimes being called "miracle leaf" for their use in treating various ailments (Akulova-Barlow, 2009; Milad et al., 2014). In the developing world, members of this genus are used for treating myriad medical conditions. Because of its widespread distribution and ubiquitous ethnobotanical use, much research has been focused on K. pinnata, a species native to Madagascar but cultivated and distributed throughout the tropics (Descoings, 2003; Biswas et al., 2011a; Quazi Majaz et al., 2011; Pattewar, 2012; Rajsekhar et al., 2016). This species has even been the subject of bioengineering - a transgenic $K$. pinnata that produces an antimicrobial peptide (AMP cecropin P1) has recently been developed (Zakharchenko et al., 2016; Lebedeva et al., 2017).

Because the genus has demonstrated medicinal potential, Kalanchoe spp. neglected in research should be explored for bioactive compounds. K. mortagei and K. fedtschenkoi, two members of the section Bryophyllum within the genus, are two such species with established ethnobotanical usage, but which have been overlooked in natural products research.

Kalanchoe mortagei, also known by the synonyms K. poincarei or Bryophyllum mortagei, is a plant native to rocky/sandy soils in north Madagascar (Descoings, 2003). Compared to other members of the genus, little research has been conducted on the chemical and medicinal properties of this species (Maiti et al., 1995). Despite this, K. mortagei is grown in Mexican homegardens, and its leaves are taken orally for digestive disorders and as a local remedy for cancer in Antioquia Department, Colombia (Blanckaert et al., 2004; Vera-Marín and Sánchez-Sáen, 2016). The roots of the plant are used for treating parasitic worm-related diseases in parts of Indonesia (Herawati and Husin, 2000).

Kalanchoe fedtschenkoi is a perennial native to central/southern Madagascar but is naturalized well outside its original range (Descoings, 2003). Introduced populations can be found in Florida, Texas, and Puerto Rico (USDA/NRCS, 2013). A popular garden succulent, K. fedtschenkoi is a model organism for research into Crassulacean acid metabolism (CAM) (Dittrich, 1976; Nimmo et al., 1986; Cook et al., 1995). In Brazil, this species is used as an analgesic (Cumberbatch, 2011).

\section{Antimicrobial Resistance in the ESKAPE Pathogens}

The rise of antimicrobial resistant (AMR) bacterial infections is one of the most pressing issues in medicine. Increasingly, conventional antibiotic medications are failing to stop persistent and dangerous bacterial diseases (Irenji et al., 2018; Katsuura et al., 2018). A report commissioned by the UK government notes that roughly 700,000 people die annually from AMR infections; this figure is projected to increase to 10 million deaths per year by 2050 (O’Neil, 2016) and encompasses data from across the broad spectrum of pathogenic microbes. In the face of rising morbidity and mortality due to AMR infections, the need for new drugs to address drug-resistance is clear (van der Meer et al., 2014). In 2015, the WHO launched the Global Antimicrobial Resistance Surveillance System (GLASS) to unify worldwide AMR. To date they have collected data from 42 countries and received over 500,000 AMR pathogenic strains (WHO, 2017).

Six bacterial species, the "ESKAPE" pathogens, have been highlighted by the Infectious Disease Society of America (IDSA) as being especially dangerous due to their patterns of antibiotic resistance. They are responsible for the majority of nosocomial infections worldwide (Table 1) (Boucher et al., 2009).

\section{Kalanchoe Spp. as a Source of Antimicrobial Treatment}

Plants used in traditional medicine are a potential source for novel antimicrobial compounds (Rahman et al., 2018; Salam and Quave, 2018). In the developing world, the large majority of people (75\%) rely on plants for primary medical needs, including for wound healing and antimicrobial agents (Sarker et al., 2005). Historically, the bulk of manufactured drugs were derived from plant natural products, and the majority of these drugs were tied directly to their original ethnobotanical use (Chin et al., 2006; Sarker and Nahar, 2012). Even between 1982 and 2002, 79\% of approved drugs worldwide had a natural product origin (Chin et al., 2006).

Secondary metabolites taken from plants used in traditional medicine have been found to inhibit microbial growth and virulence. Kalanchoe spp. have demonstrated such antimicrobial properties, and have been proven to accelerate wound-healing. For example, extracts and compounds from $K$. pinnata are effective against cutaneous leishmaniasis, a disease caused by trypanosome protozoa (Torres-Santos et al., 2003; Muzitano et al., 2006a,b, 2009).

In the past decade, substantial research has examined the antibacterial properties of K. pinnata and several other Kalanchoe spp. Extracts of K. blossfeldiana, K. crenata, K. laciniata, and $K$. pinnata have all demonstrated growth inhibitory effects on over 15 bacterial species, including four of the ESKAPE pathogens (Tables 2, 3).

In 15 studies that evaluated the antimicrobial effects of Kalanchoe spp., 12 focused solely on K. pinnata, one on 
TABLE 1 | Description of the ESKAPE pathogens.

\begin{tabular}{|c|c|c|c|}
\hline & Species & Gram & Drug development needs (Boucher et al., 2009) \\
\hline$E$ & Enterococcus faecium & + & $\begin{array}{l}\text { (VRE) Third most frequent cause of nosocomial blood borne infections. Increasing vancomycin } \\
\text { resistance. }\end{array}$ \\
\hline S & Staphylococcus aureus & + & $\begin{array}{l}\text { (MRSA) Need for oral treatment agents, less cytotoxic drugs; current drugs subject to emerging } \\
\text { resistance. Need for non-drug therapies. }\end{array}$ \\
\hline K & Klebsiella pneumoniae & - & $\begin{array}{l}\text { Can produce extended-spectrum beta-lactamases (ESBL) or are carbapenem-resistant; ESBL } \\
\text { is associated with increased mortality and delay of effective therapy. }\end{array}$ \\
\hline A & Acinetobacter baumannii & - & $\begin{array}{l}\text { Rising global incidence of infection, can be carbapenem-resistance, increased mortality for burn } \\
\text { patients. Serious absence of available treatment options. }\end{array}$ \\
\hline$P$ & Pseudomonas aeruginosa & - & Rising incidence; resistance to carbapenems, quinolones, polymyxins. \\
\hline$E$ & Enterobacter spp. & - & Rising incidence, ESBL, carbapenem-resistance. \\
\hline
\end{tabular}

K. laciniata (Iqbal et al., 2016) and one on K. blossfeldiana, a common household ornamental (Sarkar et al., 2015). A 2007 study compared the growth-inhibitory properties of $K$. crenata favorably with $K$. pinnata (Akinsulire et al., 2007).

Ten studies examined methanolic extracts, the most common solvent tested. Ethanol and water (five studies each) were also frequently used solvents. Research has established that methanolic crude extracts of $K$. pinnata outperform aqueous extracts in their growth-inhibitory effects (Akinsulire et al., 2007; Majaz et al., 2011; Nwadinigwe, 2011; Pattewar et al., 2013); this is also true for K. crenata (Akinsulire et al., 2007).

Studies also established the antibacterial effects of flavonoids extracted from K. pinnata (Okwu and Nnamdi, 2011; Tatsimo et al., 2012), as well as its leaf juice (Obaseiki-Ebor, 1985; Akinsulire et al., 2007). At least one study demonstrated the effects of $K$. pinnata in vivo, looking at how aqueous extracts accelerate the healing of wounds infected with Staphylococcus aureus and/or Pseudomonas aeruginosa (Lebedeva et al., 2017).

Research has firmly established $K$. pinnata as a plant of medicinal interest, and the overall genus continues to show promise as a potential source of antimicrobial, antibacterial compounds.

The aim of this study was to evaluate the antimicrobial potential of two previously neglected species: K. mortagei and K. fedtschenkoi against a panel of clinically relevant ESKAPE pathogens.

\section{MATERIALS AND METHODS}

\section{Plant Collection and Identification}

Two plant species were used in this experiment. Kalanchoe mortagei plants were grown from a specimen collected by the first author (NR) in Bradenton, FL, United States, in May 2008 (27.468591, -82.577127). A single K. fedtschenkoi plant was procured from the University of Georgia Plant Biology Greenhouse in Athens, GA, United States, in 2015. All plant material used in this experiment came from plants propagated from these two mother specimens. Plants were grown in NR's personal collection and at the Emory University Greenhouse. Voucher specimens of each species were deposited at the Emory University Herbarium (GEO), and species identification confirmed by Dr. Tharanga Samarakoon at
GEO (Accession nos.: 22702 and 22474 for K. fedtschenkoi and $K$. mortagei, respectively). Specimens were digitized and are available for viewing on the SERNEC portal (SERNEC, 2018).

Bulk plant materials were harvested, dried in a dehumidification chamber, and homogenized in a Waring blender into a fine powder. Retention vouchers of dried and ground material were prepared for future reference and stored in Quave Research Group laboratories at Emory University.

\section{Preparation of Extracts}

A total of seven crude extracts were prepared, four from $K$. mortagei and three from $K$. fedtschenkoi (Table 4). Each extract represented a particular plant part or combination of parts, though extract creation was also guided by limitations in available plant biomass.

Dry, ground plant biomass was double macerated for $72 \mathrm{~h}$ each with either 80 or $95 \%$ ethanol at a 1:10 ratio $(w / v)$. The extracts were agitated daily and then vacuum filtered. The aqueous extract (1509aq) was prepared as a decoction; the dry plant material was boiled with deionized water $\left(\mathrm{dH}_{2} \mathrm{O}\right)$ for $20 \mathrm{~min}$ and then filtered. After filtration the solvent was removed by rotary evaporation at $\leq 40^{\circ} \mathrm{C}$. Extracts were redissolved in $\mathrm{dH}_{2} \mathrm{O}$, shell frozen in a dry ice-acetone bath, and then lyophilized overnight on a Labconco FreeZone 2.5 Lyophilizer (Kansas City, MO, United States). Dry extracts were scraped into scintillation vials and stored at $-20^{\circ} \mathrm{C}$. Organic extracts were dissolved in DMSO and the aqueous extract was re-dissolved in $\mathrm{dH}_{2} \mathrm{O}$ to yield a stock concentration of $10 \mathrm{mg} \mathrm{mL}^{-1}$ for microbiological assays.

\section{Antibacterial Testing \\ Bacterial Strains and Cultures}

Seven extracts (Table 4) were tested against strains of ESKAPE pathogens (Table 5). Two species were Gram-positive, Enterococcus faecium (EU-44) and S. aureus (UAMS-1); the rest were Gram-negative: Klebsiella pneumoniae (CDC-16), Acinetobacter baumannii (CDC-33), P. aeruginosa (AH-71), and Enterobacter cloacae (CDC-08). Strains were streaked from freezer stock onto tryptic soy agar (TSA) plates and incubated at $37^{\circ} \mathrm{C}$ overnight. Liquid cultures in tryptic soy broth (TSB) were made from individual plate colonies in $14 \mathrm{~mL}$ test tubes and were 
TABLE 2 | Literature review of research on the antimicrobial properties of Kalanchoe spp.

\begin{tabular}{|c|c|c|}
\hline Kalanchoe sp. & Method & Microbes tested/Gram (+/-) \\
\hline $\begin{array}{l}\text { K. pinnata (Kouitcheu Mabeku et al., } \\
\text { 2017) }\end{array}$ & $\begin{array}{l}\text { Leaf methanol and ethyl acetate } \\
\text { extracts were tested against } \\
\text { Helicobacter pylori in vitro and in the } \\
\text { guts of Swiss mice. }\end{array}$ & Helicobacter pylori (-) \\
\hline $\begin{array}{l}\text { K. pinnata Transgenic and wild-type } \\
\text { (Lebedeva et al., 2017) }\end{array}$ & $\begin{array}{l}\text { Leaf aqueous extracts of wild-type and } \\
\text { transgenic (cecropin producing) were } \\
\text { applied directly to infected wounds. }\end{array}$ & $\begin{array}{l}\text { Wounds were infected with } \\
\text { Staphylococcus aureus (+), } \\
\text { Pseudomonas aeruginosa (-), or a } \\
\text { combination of both. }\end{array}$ \\
\hline K. pinnata (Larasati and Wahid, 2016) & $\begin{array}{l}\text { Leaf ethanolic extracts tested using } \\
\text { microdilution method }\end{array}$ & $\begin{array}{l}\text { Acinetobacter baumannii (-) and } \\
\text { S. aureus }(+)\end{array}$ \\
\hline K. laciniata (labal et al., 2016) & Aerial parts in a $60 \%$ methanolic extract & S. aureus $(+)$ and Bacillus subtilis (+) \\
\hline
\end{tabular}

K. blossfeldiana (Sarkar et al., 2015)

Methanolic extract evaluated against biofilm production

Leaf 95\% ethanolic, methanolic extracts $60 \%$ methanolic, aqueous extracts

K. pinnata (Tatsimo et al., 2012)

Evaluation of methanolic, ethanolic crude extracts, and extract partitions (in ethyl acetate, hexane)

Ethanolic extracts used in agar-diffusion method.

Root extracts of petroleum ether, chloroform, methanol, and water

K. pinnata (Majaz et al., 2011)

K. pinnata (Okwu and Nnamdi, 2011)

Two flavonoid compounds were

Stem extracts of methanol, water. Agar-diffusion isolated and tested directly
P. aeruginosa (-)

S. aureus (+), $P$. aeruginosa (-), Escherichia coli (-), and fungus Candida albicans

S. aureus (+), P. aeruginosa (-), Salmonella typhi (-) Fungi C. albicans, Candida parapsilosis, Cryptococcus neoformans

Bacillus megaterium (+), B. subtilis (+), $S$. aureus (+), E. coli (-), P. aeruginosa $(-)$, Shigella dysenteriae (-), S. typhi $(-)$, Vibrio cholera (-)

S. aureus (-), E. coli (-), P. aeruginosa (-) Fungus C. albicans.

$P$. aeruginosa (-), Klebsiella pneumoniae (-), E. coli (-), S. aureus (-) Fungi C. albicans and Aspergillus niger

S. typhi (-), P. aeruginosa (-), S. aureus $(+)$, Bacillus subtilis $(+)$, Fungi C. albicans and A. niger

\section{Results}

Methanol extract showed a significant anti-Helicobacter activity with MIC and $\mathrm{MBC}$ values of 32 and $256 \mathrm{\mu g} \mathrm{mL}^{-1}$, respectively. Also reduced bacterial load of gastric mucosa.

Both wild-type and transgenic extracts accelerated wound-healing and demonstrated anti-microbial effects, even in comparison to an antibiotic. Effective against both bacteria.

In assays the crude extract was found effective against $S$. aureus and B. subtilis, with MIC values of 5 and $2.5 \mathrm{mg} \mathrm{mL}^{-1}$, respectively.

Extract reduced biofilm formation and thickness reduced secretion of virulence factors. Concentrated extract destroyed biofilms.

Zones of inhibition, MICs established (30 mg for S. aureus). All extracts showed antimicrobial effects. $60 \%$ methanol extracts performed best.

Crude extracts displayed strong antibacterial and especially antifungal effects. Ethyl acetate fractions more strongly anti-microbial. An isolated flavonoid showed particularly strong effects.

Bacterial growth was inhibited by extract, expect for, S. typhi, V. cholera. Effects were strongest against $E$. coli, with a zone of inhibition of $8.2 \pm 0.22$.

Methanolic extracts most effective against all bacteria; no extracts effective against $C$. albicans.

Zones of inhibition, MICs established for all bacteria tested.

Bactericidal effects established against $B$. subtilis and $S$. aureus, with the methanolic extract showing strong effects. No effects against $P$. aeruginosa, C. albicans, and A. niger. $S$. aureus showed the lowest minimum inhibitory concentration (MIC) of $6.29 \mathrm{mg} \mathrm{mL}^{-1}$ in the methanol extract, while S. typhi showed the highest MIC of $9.98 \mathrm{mg} \mathrm{mL}^{-1}$ in the aqueous extract.

E. coli (-) ATCC 25922, P. aeruginosa

Methanol, aqueous extracts. Juice from squeezed leaves. Three solvents based on local alcoholic beverages. Agar diffusion, broth dilution methods to determine MIC.
$(-)$, K. pneumoniae (-), Shigella flexneri (-), Salmonella paratyphi (-), Citrobacter spp. (-) S. aureus (+) ATCC 25213, Enterococcus faecalis $(+)$, B. subtilis (+) Fungus C. albicans
Methanolic extracts of both species were effective against all tested, though Gram-positive bacteria were more susceptible. Aqueous extracts were less effective. K pinnata water extracts did not affect E. coli, K. pneumoniae, S. paratyphi, Citrobacter. Aqueous for either species did not affect C. albicans. Local solvents were not effective. Leaf juice extract was effective, particularly for $K$. crenata, against all except $C$. albicans. 
TABLE 2 | Literature review of research on the antimicrobial properties of Kalanchoe spp.

\begin{tabular}{|c|c|c|c|}
\hline Kalanchoe sp. & Method & Microbes tested/Gram (+/-) & Results \\
\hline K. pinnata (Ofokansi et al., 2005) & $\begin{array}{l}\text { Methanolic extracts. Agar-diffusion, } \\
\text { checkerboard. }\end{array}$ & $\begin{array}{l}\text { S. aureus (+) ATCC } 9637 \text {, } \\
\text { K. pneumonia (-), P. aeruginosa (-), } \\
\text { S. typhi }(-), \text { E. coli ATCC } 9637\end{array}$ & $\begin{array}{l}\text { MIC determined against } S \text {. aureus and } \\
\text { B. subtilis, K. pinnata demonstrated } \\
\text { synergistic antibacterial effects with } \\
\text { another plant }\end{array}$ \\
\hline K. pinnata (Akinpelu, 2000) & $\begin{array}{l}60 \% \text { methanolic extracts, tested at } \\
25 \mathrm{mg} \mathrm{mL}^{-1}\end{array}$ & $\begin{array}{l}\text { S. aureus }(+), \text { K. pneumoniae }(-), \\
\text { P. aeruginosa }(-), \text { E. coli }(-), \text { B. subtilis } \\
(-), \text { S. dysenteriae }(-), \text { C. albicans }\end{array}$ & $\begin{array}{l}\text { B. subtilis, E. coli, P. vulgaris, } \\
\text { S. dysenteriae, S. aureus were growth } \\
\text { inhibited. K. pneumoniae and } \\
\text { P. aeruginosa were not growth inhibited. }\end{array}$ \\
\hline K. pinnata (Obaseiki-Ebor, 1985) & Leaf juice extract $5 \%$ v/v tested & $\begin{array}{l}\text { S. aureus (+), Streptococcus pyogenes } \\
(+), \text { E. faecalis (+), E. coli (-), Proteus } \\
\text { spp. (-), Klebsiella spp. (+), Shigella } \\
\text { spp. (-), Salmonella spp. (-), Serratia } \\
\text { marcescens (-), and P. aeruginosa (-) }\end{array}$ & $\begin{array}{l}\text { Bactericidal effects against all } \\
\text { demonstrated. }\end{array}$ \\
\hline
\end{tabular}

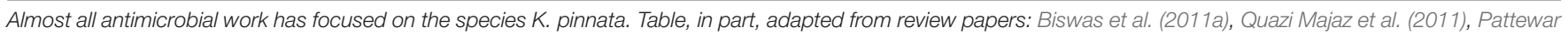
(2012), and Rajsekhar et al. (2016).

also incubated at $37^{\circ} \mathrm{C}$ overnight for use in growth inhibition assays.

\section{Growth Inhibition Assays}

The extracts were examined for the growth inhibitory activity following guidelines set by the Clinical and Laboratory Standards Institute for broth microdilution testing (CLSI, 2013). After incubation, TSB cultures were diluted in cation-adjusted Muller Hinton broth (CAMHB) based on their optical density $\left(\mathrm{OD}_{590}\right)$ to a confluence of $5 \times 10^{5} \mathrm{CFU} \mathrm{mL}^{-1}$, confirmed by plate counts. All assays were performed in CELLSTAR 96-well plates (Greiner Bio-One International, 655-185), and read in a Cytation-3 multimode plate reader (BioTek). An initial optical density reading was taken after bacterial cultures and extracts were added to each plate $\left(\mathrm{OD}_{600}\right)$. For E. faecium, S. aureus, K. pneumoniae, $P$. aeruginosa, and E. cloacae, assay plates were incubated for $18 \mathrm{~h}$; A. baumannii was incubated for $22 \mathrm{~h}$. After incubation, the optical density of wells was checked again $\left(\mathrm{OD}_{600}\right)$.

In the initial screen, each extract was tested at a concentration of $256 \mu \mathrm{g} \mathrm{mL}^{-1}$ to determine if any growth-inhibitory effects at a level of $50 \%$ or greater were evident in comparison to the vehicle (DMSO) control. If bacterial growth was inhibited by at least $50 \%$, microdilution assays were performed. Dose response studies were performed on bacteria-extract pairs exhibiting $\geq 50 \%$ growth inhibition in this initial screen. Extracts were tested by twofold serial dilution at a concentration range of 8-256 $\mu \mathrm{g} \mathrm{mL}^{-1}$.

Percent inhibition was calculated in order to minimize the influence of any color cast due to the plant extracts as previously described (Quave et al., 2008). The $\mathrm{IC}_{50}$ values were defined as the concentration required to achieve a $50 \%$ inhibition of growth, and the MIC values (or $\mathrm{IC}_{90}$ ) were defined as the concentration required to achieve $90 \%$ growth inhibition (as determined by $\mathrm{OD}_{600}$ for both values). Gentamicin was used as a positive control against all strains.

\section{Mammalian Cytotoxicity Assay}

Mammalian cytotoxicity of extracts was assessed using human keratinocytes (HaCaTs) and a lactate dehydrogenase (LDH) test kit (G-Biosciences, St. Louis, MO, United States) as previously described (Quave et al., 2015). Briefly, HaCaTs were maintained in Dulbecco's modified Eagle's medium with L-glutamine and glucose supplemented with $10 \%$ heat-inactivated fetal bovine serum and $1 \times$ solution of penicillin and streptomycin at $37^{\circ} \mathrm{C}, 5 \% \mathrm{CO}_{2}$ in $75 \mathrm{~mL}$ flasks. Once $90-95 \%$ confluency was reached, the cells were detached from the flask bottom using $0.25 \%$ trypsin, $0.1 \%$ ethylenediaminetetraacetic acid (EDTA) in Hanks' balanced salt solution (HBSS) without $\mathrm{Ca}^{++}, \mathrm{Mg}^{++}$, and $\mathrm{NaHCO}_{3}$. The culture was standardized to $4 \times 10^{4}$ cells $\mathrm{mL}^{-1}$ using a hemocytometer. Then, $200 \mu \mathrm{L}$ of the standardized culture was added to each well in a 96-well tissue culturetreated microtiter plate (Falcon 35-3075) and the plates were incubated for $48 \mathrm{~h}$ in a humidified $37^{\circ} \mathrm{C}, 5 \% \mathrm{CO}_{2}$ incubator, prior to media aspiration. Either media containing extracts (4$512 \mu \mathrm{g} \mathrm{mL}^{-1}$ ) or vehicle were serially diluted and processed $24 \mathrm{~h}$ later following manufacturer's protocol for chemical induced cytotoxicity. Percent DMSO (v/v) in the wells was $<2 \%$ for all tests.

\section{Chemical Characterization}

Each extract was characterized by HPLC using a method adapted from four previously published HPLC methods, one examining flavonoid compounds (Nielsen et al., 2005), and three examining bufadienolides (a type of cardiac glycoside commonly found in Kalanchoe plants) (Supratman et al., 2000; Huang et al., 2013; Moniuszko-Szajwaj et al., 2016). Extracts were dissolved in methanol $(1465,1469)$, methanol: $\mathrm{dH}_{2} \mathrm{O}(1420,1421$, 1509aq), or methanol: $\mathrm{dH}_{2} \mathrm{O}: \mathrm{DMSO}(1468,1508)$. All extracts were chromatographed on an Agilent 1260 Infinity system running OpenLab CDS ChemStation (Agilent Technologies, Santa Clara, CA, United States) with an Agilent ZORBAX Eclipse XDB-C18 $(250 \mathrm{~mm} \times 4.6 \mathrm{~mm}, 5 \mu \mathrm{m})$ column with compatible guard column at $30^{\circ} \mathrm{C}$. A $10 \mu \mathrm{L}$ injection of each extract was eluted at a flow rate of $1 \mathrm{~mL} \mathrm{~min}^{-1}$ using a mobile phase consisting of (A) $0.1 \%$ formic acid in $\mathrm{H}_{2} \mathrm{O}$ and (B) $0.1 \%$ formic acid in methanol (VWR HiPerSolv CHROMANORM). The gradient profile consisted of initial conditions 98:2 A:B which were held for $20 \mathrm{~min}$, 
TABLE 3 | Review of Kalanchoe extracts tested against selected bacteria in previous research.

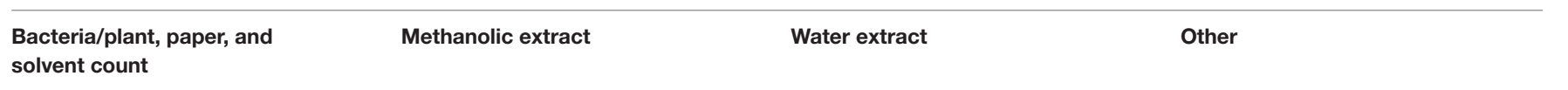

Acinetobacter baumannii (-)

K. pinnata: 1 paper, 1 extract

solvent

Bacillus subtilis (+) K. pinnata,

K. laciniata: 4 papers, 3 extract solvents

Enterobacter spp. (-)

K. pinnata: 1 paper examining

organic acid extract

Enterococcus faecalis (+)

K. pinnata, K. crenata: 2

papers, 2 solvents, and leaf juice.

Enterococcus faecium (+)

Escherichia coli (-) K. pinnata,

K. crenata: 9 papers, 5 extract

solvents, leaf juice, and

flavonoid compounds

Helicobacter pylori (-)

K. pinnata: 1 paper, 2 solvents

\section{Klebsiella pneumoniae (-)}

K. pinnata, K. crenata: 5 papers, 2 solvents, leaf juice, and flavonoid compounds

Pseudomonas aeruginosa (-) K. pinnata, K. crenata, K. blossfeldiana: 11 papers, 6 solvents, leaf juice, and flavonoid compounds

Proteus spp. (-) K. pinnata: 1 paper testing leaf juice

Salmonella typhi (-) K. pinnata: 5 papers, 5 solvents, and leaf juice

\author{
K. pinnata \\ $\checkmark+$ (Akinpelu, 2000; \\ Nwadinigwe, 2011) \\ K. laciniata \\ $\checkmark$ (lqbal et al., 2016)
}

K. pinnata

- Ethanol $\checkmark$ (Larasati and Wahid, 2016)

K. pinnata

- Ethanol $\checkmark$ (Biswas et al., 2011b)
K. pinnata $\checkmark$ (Akinsulire et al., 2007) K. crenata $\checkmark$ (Akinsulire et al., 2007)
K. pinnata
$\checkmark$ (Nwadinigwe, 2011)

\section{K. pinnata}

- Malic acid $\checkmark$ extracted from plant using decoction method, successful against E. aerogenes (Jazul, 1995)

K. pinnata

- Leaf juicer (Obaseiki-Ebor, 1985;

Akinsulire et al., 2007) K. crenata

- Leaf juicer (Akinsulire et al., 2007)

\section{K. pinnata}

$\checkmark+$ (Akinpelu, 2000; Ofokansi et al., 2005; Akinsulire et al.,

2007; Majaz et al., 2011;

Nwadinigwe, 2011) K. crenata

$\checkmark+($ Akinsulire et al., 2007)

\section{K. pinnata}

$\checkmark+$ (Kouitcheu Mabeku et al., 2017)

\section{K. pinnata}

$\checkmark+$ (Ofokansi et al., 2005;

Akinsulire et al., 2007)

$\chi$ (Akinpelu, 2000)

K. crenata

$\checkmark+$ (Akinsulire et al., 2007)

\section{K. pinnata}

$\checkmark+$ (Ofokansi et al., 2005; Akinsulire et al., 2007; Majaz

et al., 2011; Pattewar et al.,

2013) $\chi$ (Akinpelu, 2000;

Nwadinigwe, 2011)

K. crenata

$\checkmark+$ (Akinsulire et al., 2007)

K. blossfeldiana

$\checkmark$ (Sarkar et al., 2015) inhibited

biofilm production**

No Kalanchoe extracts previously tested against this species

\section{K. pinnata}

$\checkmark$ (Akinsulire et al., 2007; Majaz

et al., 2011; Nwadinigwe,

2011; Pattewar et al., 2013)

$\chi$ (Akinsulire et al., 2007)

K. crenata

$\checkmark$ (Akinsulire et al., 2007)
K. pinnata $\chi$ (Akinsulire et al., 2007)

K. crenata

$\checkmark$ (Akinsulire et al., 2007)

\section{K. pinnata}

$\checkmark$ (Akinsulire et al., 2007; Majaz et al., 2011; Nwadinigwe,

2011; Pattewar et al., 2013)

K. crenata

$\checkmark$ (Akinsulire et al., 2007)
K. pinnata $\checkmark+$ (Ofokansi et al., 2005; Nwadinigwe, 2011; Tatsimo et al., 2012) K. pinnata $\chi$ (Nwadinigwe,
2011)

\section{K. pinnata}

- Ethanol $\checkmark$ (Biswas et al., 2011b;

Pattewar et al., 2013)

- Petroleum ether, chloroform* (Majaz et al., 2011)

- Flavonoid compounds $\checkmark$ (Okwu and Nnamdi, 2011)

- Leaf juice $\checkmark$ (Obaseiki-Ebor, 1985; Akinsulire et al., 2007)

\section{K. crenata}

- Leaf juice $\checkmark$ (Akinsulire et al., 2007)

K. pinnata

- Ethyl acetate $\chi$ (Kouitcheu Mabeku et al., 2017)

\section{K. pinnata}

- Flavonoid compounds $\checkmark$ (Okwu and Nnamdi, 2011)

- Leaf juice $\checkmark$ (Obaseiki-Ebor, 1985; Akinsulire et al., 2007) K. crenata - Leaf juice $\checkmark$ (Akinsulire et al., 2007)

\section{K. pinnata}

- Ethanol $\checkmark$ (Biswas et al., 2011b; Tatsimo et al., 2012; Pattewar et al., 2013)

- Ethyl acetate $\checkmark+$, hexane $\chi$ fractions

(Tatsimo et al., 2012)

- Petroleum ether, chloroform* (Majaz et al., 2011)

- Flavonoid compounds (Okwu and Nnamdi, 2011) $\checkmark$

- Leaf juice $\checkmark$ (Obaseiki-Ebor, 1985;

Akinsulire et al., 2007) K. crenata

- Leaf juicer (Akinsulire et al., 2007)

K. pinnata

- Leaf juice $\checkmark$ (Obaseiki-Ebor, 1985)

K. pinnata

- Ethanolic (Biswas et al., 2011b;

Tatsimo et al., 2012)

- Ethyl acetate $\checkmark+$, hexane $\chi$ fractions

(Tatsimo et al., 2012)

- Leaf juice (on Salmonella spp.)

(Obaseiki-Ebor, 1985) 
TABLE 3 | Continued

\begin{tabular}{|c|c|c|}
\hline $\begin{array}{l}\text { Bacteria/plant, paper, and } \\
\text { solvent count }\end{array}$ & Methanolic extract & Water extract \\
\hline $\begin{array}{l}\text { Shigella dysenteriae (-) } \\
\text { K. pinnata: } 3 \text { papers, } 2 \\
\text { solvents, and leaf juice. }\end{array}$ & K. pinnata $\checkmark($ Akinpelu, 2000) & \\
\hline $\begin{array}{l}\text { Staphylococcus aureus (+) } \\
\text { K. pinnata, K. crenata: } 14 \\
\text { papers, } 7 \text { solvents, leaf juice, } \\
\text { and flavonoid compounds }\end{array}$ & $\begin{array}{l}\text { K. pinnata } \checkmark+\text { (Akinpelu, } \\
\text { 2000; Ofokansi et al., 2005; } \\
\text { Majaz et al., 2011; } \\
\text { Nwadinigwe, 2011; Tatsimo } \\
\text { et al., 2012; Pattewar et al., } \\
\text { 2013; Lebedeva et al., 2017) } \\
\text { K. crenata } \checkmark+\text { (Akinsulire } \\
\text { et al., 2007) K. laciniata (labal } \\
\text { et al., 2016) }\end{array}$ & $\begin{array}{l}\text { K. pinnata } \checkmark \text { (Akinsulire et al., } \\
\text { 2007; Majaz et al., 2011; } \\
\text { Nwadinigwe, 2011; Pattewar } \\
\text { et al., 2013) K. crenata } \checkmark \\
\text { (Akinsulire et al., 2007) }\end{array}$ \\
\hline
\end{tabular}

Serratia marcescens (-)

K. pinnata: 1 paper testing leaf

juice

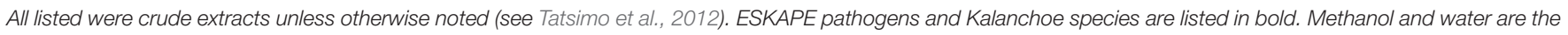

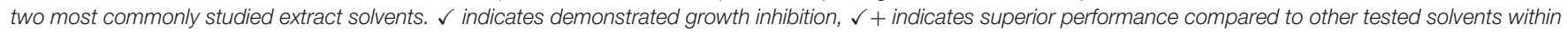
the same study. $\chi$ indicates no significant growth inhibition demonstrated. Next to species name, bacteria are noted as Gram positive (+) or negative (-).

TABLE 4 | Extracts of $K$. mortagei and $K$. fedtschenkoi used in this study.

\begin{tabular}{|c|c|c|c|c|c|}
\hline Extract number & $\begin{array}{l}\text { Species } \\
\text { extracted }\end{array}$ & Plant part extracted & $\begin{array}{c}\text { Extraction } \\
\text { solvent }\end{array}$ & Yield (\%) & $\begin{array}{c}\text { Total phenolic } \\
\text { content (mg GAE/g) }\end{array}$ \\
\hline 1420 & K. mortagei & $\begin{array}{l}\text { Leaves, stems (aerial parts), } \\
\text { immature inflorescences }\end{array}$ & $80 \% \mathrm{EtOH}$ & 19.62 & $331 \pm 33$ \\
\hline 1468 & K. mortagei & Leaves, stems (aerial parts) & $95 \% \mathrm{EtOH}$ & 6.98 & $571 \pm 87$ \\
\hline 1508 & K. mortagei & Mature inflorescence, flowers & $95 \% \mathrm{EtOH}$ & 16.13 & $818 \pm 19$ \\
\hline 1509aq & K. mortagei & Mature inflorescence, flowers & $\mathrm{H}_{2} \mathrm{O}$ & 22.25 & $1340 \pm 116$ \\
\hline 1421 & K. fedtschenkoi & $\begin{array}{l}\text { Aerial parts (including woody } \\
\text { stems) }\end{array}$ & $80 \% \mathrm{EtOH}$ & 12.69 & $370 \pm 17$ \\
\hline 1465 & K. fedtschenkoi & Woody stems & $95 \% \mathrm{EtOH}$ & 7.44 & $498 \pm 50$ \\
\hline 1469 & K. fedtschenkoi & Aerial parts (no woody stems) & $95 \% \mathrm{EtOH}$ & 15.54 & $486 \pm 6$ \\
\hline
\end{tabular}

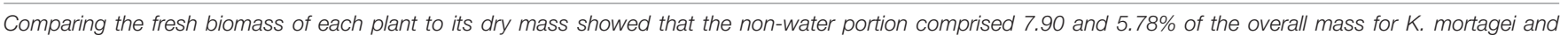
K. fedtschenkoi, respectively. The TPC is expressed in mg GAE/g dry extract.

then increased to $24.5: 75.5 \mathrm{~A}: \mathrm{B}$ from 20 to $95.5 \mathrm{~min}$, and finally to $100 \% \mathrm{~B}$ at $110 \mathrm{~min}$, which was held for $20 \mathrm{~min}$. Chromatograms of each extract were generated using ultravioletvisual spectroscopy (UV-vis) during HPLC, and reported at $254 \mathrm{~nm}$.

Standard flavonoids, kaempferol (MP Biomedicals, Inc.), and quercetin (Enzo Life Sciences), as well as phenolic compounds, caffeic acid, $p$-coumaric acid, and ferulic acid (MP Biomedicals, Inc.) were used to aid in characterization by HPLC.

\section{Detection of Total Phenolic Content}

Total phenolic content (TPC) was determined using a FolinCiocalteu assay modified for 96-well plate format (Singleton et al., 1999). A $1 \mathrm{mg} \mathrm{mL} \mathrm{m}^{-1}$ gallic acid stock solution was prepared in $50 \% \mathrm{MeOH}_{(\mathrm{aq})}$ and diluted in the same solution to yield
0-100 $\mu \mathrm{g} \mathrm{mL}^{-1}$ gallic acid standard solutions. Extracts were prepared at 1 or $2 \mathrm{mg} \mathrm{mL}^{-1}$ in $50 \% \mathrm{MeOH}_{(\mathrm{aq})}$ and serially diluted until their absorbance was within the range of the gallic acid standard curve. In a 96-well plate, $30 \mu \mathrm{L}$ of gallic acid standard solution or extract was added to triplicate wells. To each well $200 \mu \mathrm{L}$ of $\mathrm{dH} 2 \mathrm{O}$ was added, then $15 \mu \mathrm{L}$ of FolinCiocalteu regent. After at least $1 \mathrm{~min}$, but no more than $8 \mathrm{~min}$, $50 \mu \mathrm{L}$ of $20 \% \mathrm{Na}_{2} \mathrm{CO}_{3}(\mathrm{w} / \mathrm{v})$ was added to all wells. The plate was mixed for $30 \mathrm{~s}$ on an orbital shaker, incubated at $40^{\circ} \mathrm{C}$ for $30 \mathrm{~min}$, manually mixed with a multichannel pipette, then an additional $30 \mathrm{~s}$ with an orbital shaker, and finally the absorbance at $760 \mathrm{~nm}$ was recorded using a BioTek Cytation 3 multimode plate reader. The linear range for the assay was determined as $0-100 \mu \mathrm{g} \mathrm{mL}^{-1}$ gallic acid equivalents (GAE), $R^{2}=0.986$. The TPC of the extracts is expressed as mg GAE/g dry extract. 
TABLE 5 | ESKAPE pathogens tested and their corresponding antibiotic resistance profiles as reported by the source provider (BEI Resources or CDC AR Bank) or as determined by antibiotic disc diffusion test (for AMC, IPM, PIP, RA, SXT, and TET) following CLSI breakpoints.

\begin{tabular}{|c|c|c|c|c|}
\hline Species & Strain ID & Alternate ID & Antibiotic resistance profile* & Other characteristics \\
\hline Enterococcus faecium & EU-44 & HM-959; Strain 513 & AMC, RIF, SXT, TET, TZP & \\
\hline Staphylococcus aureus & UAMS-1 & & & $\begin{array}{l}\text { Osteomyelitis isolate; MSSA; } \\
\text { prototype biofilm isolate }\end{array}$ \\
\hline Klebsiella pneumoniae & CDC-16 & AR-Bank \#0016 & AMP, ATM', FOX, SAM', TET & $\begin{array}{l}\text { Reduced susceptibility, } \\
\text { elevated carbapenem MICs }\end{array}$ \\
\hline Acinetobacter baumannii & CDC-33 & AR-Bank \#0033 & $\begin{array}{l}\text { CAZ, CIP, CRO, CTX, DOR, FEP, } \\
\text { GEN, IPM, LVX, MEM, SAM, SXT, } \\
\text { TOB, TZP }\end{array}$ & $\begin{array}{l}\text { Reduced susceptibility, } \\
\text { elevated carbapenem MICs }\end{array}$ \\
\hline Pseudomonas aeruginosa & $\mathrm{AH}-0071$ & $\mathrm{PAO} 1$ & & \\
\hline Enterobacter cloacae & CDC-08 & AR-Bank \#0008 & $\begin{array}{l}\text { AMC, AMP, ATM, CAZ, CFZ, CIP, } \\
\text { CRO, CTX, DOR', ETP, FOX, LVX, } \\
\text { MEM', SAM, TET, TZP }\end{array}$ & $\begin{array}{l}\text { Reduced susceptibility, } \\
\text { elevated carbapenem MICs }\end{array}$ \\
\hline
\end{tabular}

*Resistance: AMC, amoxicillin-clavulanic acid; AMP, ampicillin; ATM, aztreonam; CAZ, ceftazidime; CIP, ciprofloxacin; CRO, ceftriaxone; CTX, cefotaxime; DOR, doripenem; ETP, ertapenem; FEP, cefepime; FOX, cefoxitin; GEN, gentamicin; IPM, imipenem; LVX, levofloxacin; MEM, meropenem; RIF, rifampicin; SAM, ampicillinsublactam; SXT, trimethoprim-sulfamethoxazole; TET, tetracycline; TOB, tobramycin; TZP, piperacillin-tazobactam. Any antibiotics denoted with I indicates resistance.

TABLE 6 | Extracts exhibiting $I_{50}$ growth inhibition ( $\left.\geq 50 \%\right)$ against ESKAPE pathogens.

\begin{tabular}{|c|c|c|c|c|c|c|c|}
\hline & & E. faecium & S. aureus & K. pneumoniae & A. baumannii & P. aeruginosa & E. cloacae \\
\hline Species & Extract ID & EU-44 & UAMS-1 & CDC-16 & CDC-33 & AH-71 & CDC-08 \\
\hline \multirow[t]{4}{*}{ K. mortagei } & 1420 & $>256$ & $>256$ & $>256$ & $>256$ & $>256$ & $>256$ \\
\hline & 1468 & $>256$ & $>256$ & $>256$ & $>256$ & $>256$ & $>256$ \\
\hline & 1508 & $>256$ & $>256$ & $>256$ & $>256$ & $>256$ & $>256$ \\
\hline & $1509 a q$ & $>256$ & $>256$ & $>256$ & $>256$ & $>256$ & $>256$ \\
\hline \multirow[t]{3}{*}{ K. fedtschenkoi } & 1421 & $>256$ & $>256$ & $>256$ & 256 & $>256$ & $>256$ \\
\hline & 1465 & $>256$ & 256 & $>256$ & 128 & 128 & $>256$ \\
\hline & 1469 & $>256$ & 256 & $>256$ & 256 & 256 & $>256$ \\
\hline Gentamicin MIC & & 8 & 16 & $>64$ & $>64$ & 4 & $<4$ \\
\hline
\end{tabular}

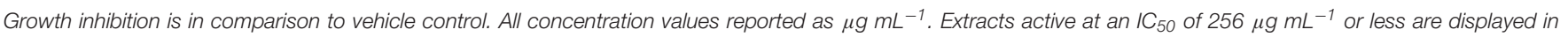
bold.

\section{RESULTS}

\section{K. fedtschenkoi Exhibits Antibacterial Activity Against Three ESKAPE Pathogens}

Initial screening of extracts at $256 \mu \mathrm{g} \mathrm{mL}^{-1}$ demonstrated an $\mathrm{IC}_{50}$ (growth inhibition of $50 \%$ or greater) of $\mathrm{K}$. fedtschenkoi extracts $(1421,1465$, and 1469) against three of the ESKAPE pathogens: S. aureus, A. baumannii, and P. aeruginosa. Further testing by serial dilution assays revealed that $K$. fedtschenkoi extracts had $\mathrm{IC}_{50}$ values ranging from 128 to $256 \mu \mathrm{g} \mathrm{mL}{ }^{-1}$ for these pathogens (Table 6).

Growth inhibition by dose response is reported in Figure 1. The only extract to exhibit $>35 \%$ inhibition in E. faecium (EU-44) was 1468 . No extracts inhibited growth of E. cloacae (CDC-08) by $20 \%$ or more. Extract 1465 (K. fedtschenkoi woody stems) exhibited $>60 \%$ inhibition in A. baumannii (CDC-33) and an MIC of $256 \mu \mathrm{g} \mathrm{mL}^{-1}$ (growth inhibition $\geq 90 \%$ ) was observed against $P$. aeruginosa (AH-71). Extract 1508 was the only extract to exhibit at least $40 \%$ inhibition in growth at $256 \mu \mathrm{g} \mathrm{mL}-1$ against $K$. pneumoniae.

\section{Extracts Exhibit Low Toxicity to Human Keratinocytes}

Human skin keratinocytes (HaCaTs) were exposed to each extract to examine possible cytotoxic effects in mammalian cells. The highest levels of cytotoxicity were observed at the $512 \mu \mathrm{g} \mathrm{mL} \mathrm{mL}^{-1}$ concentration, and ranged from 11 to $26 \%$ growth inhibition of human cells. All extracts at the $256 \mu \mathrm{g} \mathrm{mL}^{-1}$ concentration exhibited cytotoxicity of $12 \%$ or less (Figure 2). No $\mathrm{IC}_{50}$ was observed for any of the tested concentrations.

\section{Chemical Characterization of Extracts}

In this study, the Kalanchoe spp. extracts were screened by HPLC for the presence of several commonly occurring flavonoids; kaempferol (1) and quercetin (2), and phenolic compounds, caffeic acid (3), p-coumaric acid (4), ferulic acid (5). Both K. mortagei (extracts 1421 and 1469) and K. fedtschenkoi (extract 1468) contained 2. The extracts 1421 and 1465 of K. fedtschenkoi contained $\mathbf{1}$. The presence of $\mathbf{3}$ was also established in K. fedtschenkoi (extracts 1421 and 1465) and in K. mortagei (extract 1468) at very low levels (Figure 3). 

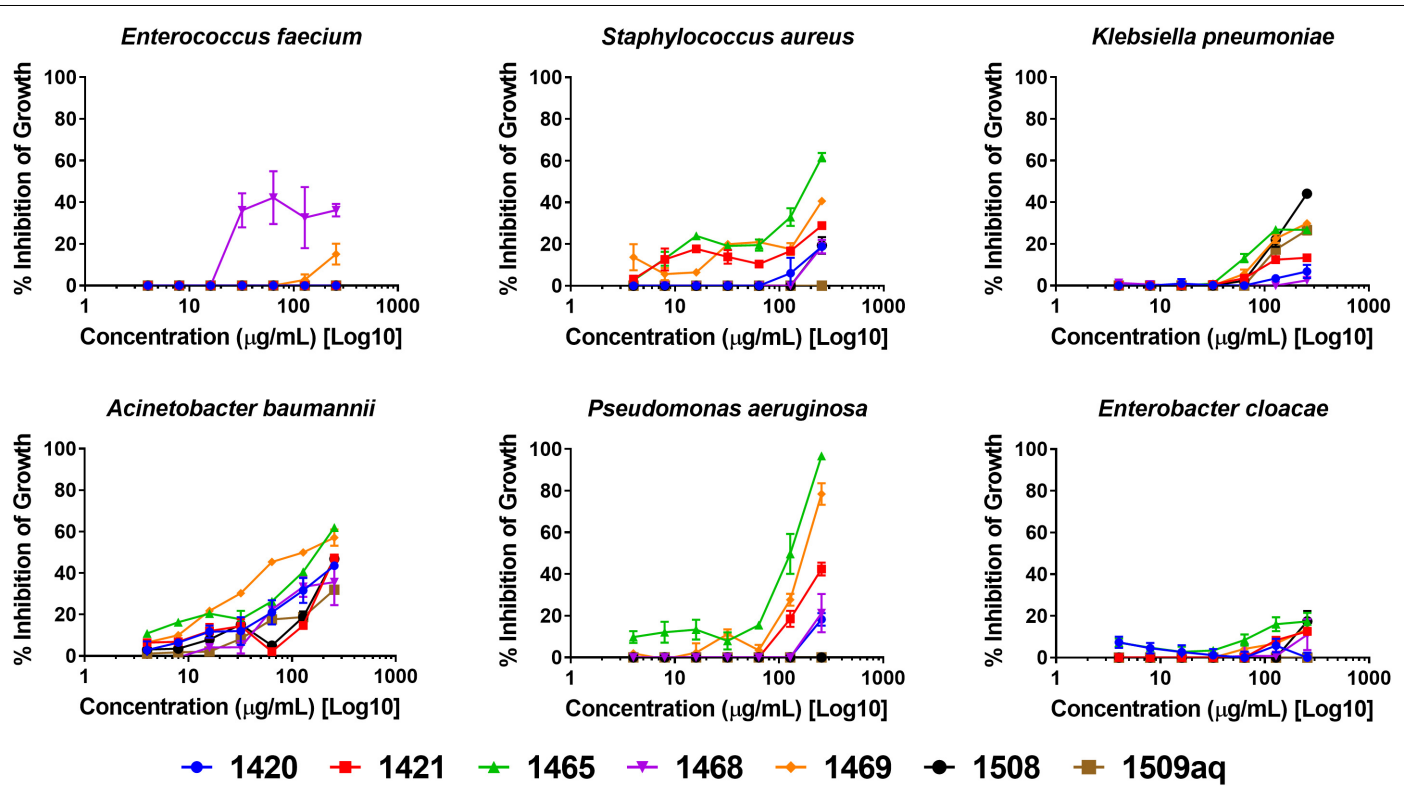

FIGURE 1 | Growth inhibitory activity of Kalanchoe spp. extracts.

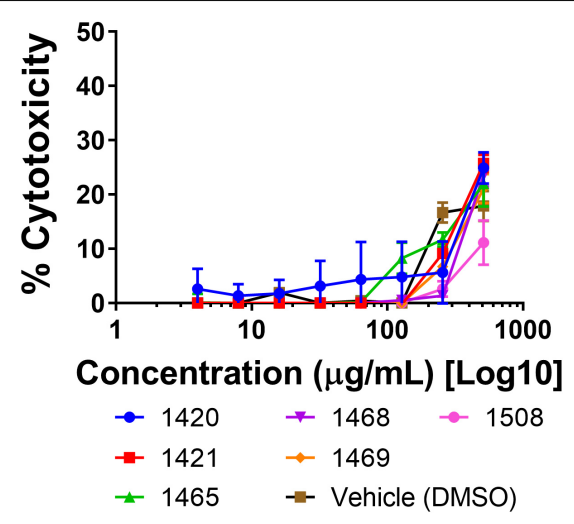

FIGURE 2 | Cytotoxicity of extracts in a human keratinocyte ( $\mathrm{HaCaT}$ ) cell line by LDH assay for cell viability.

Previous studies have shown a diverse chemistry in the genus Kalanchoe. Previous studies identified $\mathbf{5}$ in several Kalanchoe species (Gaind and Gupta, 1971; Muzitano et al., 2006a,b; Cruz et al., 2012). 1 was established in K. pinnata (Gaind and Gupta, 1971; Muzitano et al., 2006a) and K. daigremontiana (Ürményi et al., 2016). Syringic acid, $\mathbf{3}$, and $\mathbf{4}$ were identified in K. pinnata (Gaind and Gupta, 1973). A 1995 study found lupeol, lupeol acetate, $\beta$-sitosterol, and other related compounds in K. mortagei (Maiti et al., 1995).

The TFC for the Kalanchoe spp. extracts ranged from a minimum of $331 \pm 33 \mathrm{mg}$ GAE/g extract for 1420 to $1340 \pm 116 \mathrm{mg}$ GAE/g extract for 1509aq. The K. mortagei inflorescences extracts (1508 and 1509aq) had higher TFC than the other plant parts of both species, $818 \mathrm{mg}$ GAE/g extract and $1340 \mathrm{mg}$ GAE/g extract, respectively. The average TFC of the K. mortagei extracts with leaf and stem tissues (1420 and 1468) and the K. fedtschenkoi leaf and stem tissue extracts (1420 and 1465) were both $451 \mathrm{mg}$ GAE/g extract, indicating that both species have similar TFC. However, the K. fedtschenkoi leaf and stem tissue extracts (1421, 1465, and 1469) have higher antimicrobial activities against multiple bacterial strains than the $K$. mortagei extracts. This suggests that the bioactivity of $K$. fedtschenkoi is not due to phenolic compounds.

\section{DISCUSSION}

In this study, K. fedtschenkoi extracts exhibited growth inhibition against two Gram-negative species, A. baumannii (CDC-33) and $P$. aeruginosa (AH-71), as well as Gram-positive S. aureus. All other pathogens examined, including Gram-positive E. faecium (EU-44), were largely unaffected. This contrasts with some previous work, where Kalanchoe spp. extracts tested against bacteria exhibited growth-inhibitory effects more readily against Gram-positive pathogens (Akinsulire et al., 2007). Extracts in other studies with $S$. aureus have always shown growthinhibition, with the exception of the poor-performance of a hexane fraction tested (Tatsimo et al., 2012; Table 3). Tests against Gram-negative species $P$. aeruginosa and K. pneumoniae have had mixed results, demonstrating both positive (Akinsulire et al., 2007; Pattewar et al., 2013) and negative (Akinpelu, 2000; Nwadinigwe, 2011) results concerning growth-inhibition.

Although K. mortagei extracts 1420 and 1468 failed to inhibit growth at or above $50 \%\left(\mathrm{IC}_{50}\right)$, there were differences in performance and chemical characterization of these two closely related extracts. Both 1420 and 1468 were composed of aerial parts of $K$. mortagei (leaves and stems), though 1420 also had immature inflorescences. Against E. faecium (EU-44) and 


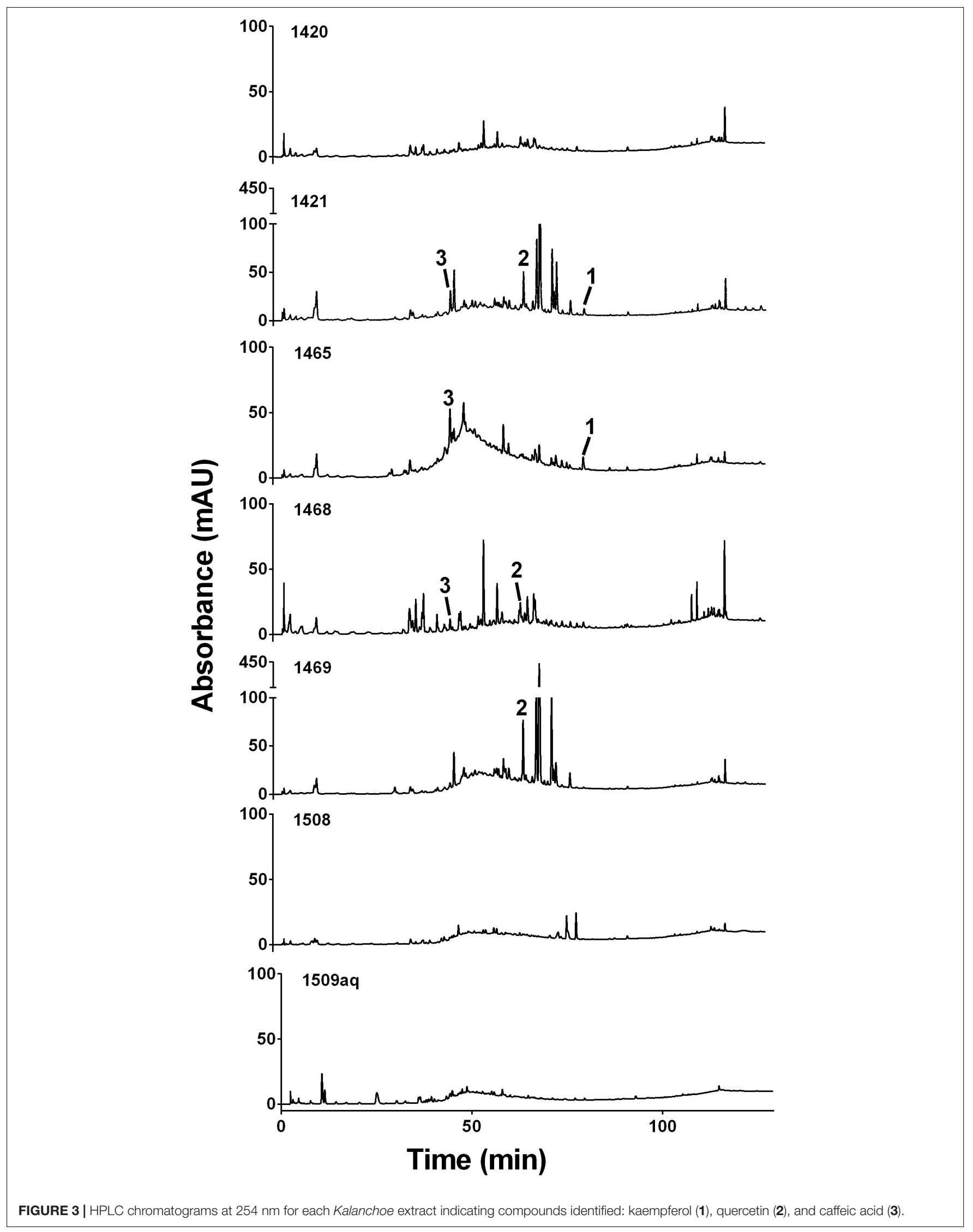


P. aeruginosa (AH-71), 1420 actually increased bacterial growth, and against all six pathogens, there were statistically different performances between these two extracts (verified with Student's $t$-test). HPLC analysis revealed lower absorbance intensity in the 35-80 min region for 1420 compared to 1468 , though elution peaks were similar. Caffeic acid could only be confirmed in 1420, and kaempferol was only confirmed in 1468 .

It is possible that the differences are due to the harvest conditions of the $K$. mortagei plants used to make these extracts. Extract 1420 was prepared from a K. mortagei plant collected in December 2017, which was maintained in low-light conditions. Extract 1468, in contrast, was collected in March 2018 and was grown in bright light in a greenhouse setting. Research has shown that the chemical composition of $K$. pinnata is dependent on the plant's light, growth, and harvest conditions; in bright light, the concentration of quercetin increased sevenfold, and that flavonoid compounds were more abundant during summer months (Muzitano et al., 2011). It is possible that the suboptimal growth conditions of the $K$. mortagei plant used for extract 1420 prevented the production of certain bioactive secondary metabolites.

\section{CONCLUSION}

Kalanchoe is an important genus with relevance to traditional medicine across the globe. We have provided a comprehensive review of the reported antibacterial activities of Kalanchoe species, in particular K. pinnata, K. crenata, K. blossfeldiana, and $K$. laciniata. For the first time, we have reported the antibacterial activities of two understudied species in this genus ( $K$. fedtschenkoi and $K$. mortagei) against clinically relevant, multidrug-resistant (MDR) strains of Gram-positive and Gram-negative bacteria. Our counterscreens against $\mathrm{HaCaTs}$ demonstrated that these extracts exhibit low toxicity to mammalian cells, supporting specificity of the action of these extracts against bacterial pathogens. Extracts were also characterized by HPLC, using chemical standards for peak identification and differentiation in their composition.

We demonstrated the antibacterial potential of K. fedtschenkoi against three ESKAPE pathogens. Particularly noteworthy was the specific growth-inhibition observed for A. baumannii, a Gram-negative species with rising global incidence that currently lacks sufficient treatment options (Boucher et al., 2009). In order to fully examine the potential of $K$. fedtschenkoi secondary metabolites, future work should aim to characterize the bioactivity of different extracts through bioassay-guided fractionation and isolation of active fractions and/or individual

\section{REFERENCES}

Akinpelu, D. A. (2000). Antimicrobial activity of Bryophyllum pinnatum leaves. Fitoterapia 71, 193-194. doi: 10.1016/S0367-326X(99)00135-5

Akinsulire, O. R., Aibin, I., Adenipekun, T., Adelowotan, T., and Odugbemi, T. (2007). In vitro antimicrobial activity of crude extracts from plants Bryophyllum pinnatum and Kalanchoe crenata. Afr. J. Tradit. Complement. Altern. Med. 4, 338-344. doi: 10.4314/ajtcam.v4i3.31227 compounds. Additional studies should also look to address potential biofilm-inhibitory properties and interference in bacterial quorum sensing. $K$. blossfeldiana extracts have been shown to reduce biofilm growth or destroy biofilms entirely (Sarkar et al., 2015), and biofilm inhibition remains one of the most likely avenues for successful implementation of an anti-bacterial agent derived from plants (Wright, 2017). Other members of the genus Kalanchoe neglected in research should also be assessed for anti-microbial potential.

\section{AUTHOR CONTRIBUTIONS}

NR grew and collected the plant specimens, prepared the extracts, and performed the antibacterial experiments. JL and NR performed the chemical analysis of the extracts. BD performed the $\mathrm{HaCaT}$ cytotoxicity experiments. CQ designed and directed the study. NR and CQ analyzed the data and wrote the manuscript. All authors read, revised, and approved the final manuscript.

\section{FUNDING}

This work was supported by the National Institutes of Health, National Institute of Allergy and Infectious Disease (R21 AI136563, PI: CQ). The content is solely the responsibility of the authors and does not necessarily reflect the official views of the NIH or NIAID. The funding agency had no role in study design, data collection and analysis, decision to publish, or preparation of the manuscript.

\section{ACKNOWLEDGMENTS}

The following reagent was obtained through BEI Resources, NIAID, NIH as part of the Human Microbiome Project: E. faecium, Strain 513, HM-959. The following reagents were obtained through the Centers for Disease Control Antibiotic/Antimicrobial Resistance Bank: A. baumannii, ARBANK \#0033; E. cloacae, AR-Bank \#0008; and K. pneumoniae, AR-Bank \#0016. Thanks to Dr. Alex Horswill (UC Denver) for provision of the $P$. aeruginosa isolate $(\mathrm{AH}-71$; PAO1) and Dr. Mark Smeltzer (UAMS) for provision of the $S$. aureus isolate (UAMS-1). Thanks to Dr. Tharanga Samarakoon (Emory Herbarium) for assistance with identification of the study species and Erik Edwards (Emory Greenhouse) for assistance with sample cultivation. Succulent J. 81, 268-276. doi: 10.2985/015.081.0601

Biswas, S. K., Chowdhury, A., Das, J., Hosen, S. Z., Uddin, R., and Rahaman, M. S. (2011a). Literature review on pharmacological potentials of Kalanchoe pinnata (Crassulaceae). Afr. J. Pharm. Pharmacol. 5, 1258-1262. doi: 10.5897/AJPP 11.273

Biswas, S. K., Chowdhury, A., Das, J., Karmakar, U. K., and Shill, M. C. (2011b). Assessment of cytotoxicity and antibacterial activities of ethanolic extracts of 
Kalanchoe pinnata Linn. (family: Crassulaceae) leaves and stems. Int. J. Pharm. Sci. Res. 2:2605.

Blanckaert, I., Swennen, R. L., Flores, M. P., López, R. R., and Saade, R. L. (2004). Floristic composition, plant uses and management practices in homegardens of san rafael coxcatlán, valley of tehuacán-cuicatlán, mexico. J. Arid Environ. 57, 179-202. doi: 10.1016/S0140-1963(03)00100-9

Botha, C. (2013). Cardiac Glycoside Intoxication. The African Veterinary Information Portal. Pretoria: University of Pretoria.

Botha, C. J. (2013). Krimpsiekte in South Africa: historical perspectives. J. S. Afr. Vet. Assoc. 84:a1059. doi: 10.4102/jsava.v84i1.1059

Boucher, H. W., Talbot, G. H., Bradley, J. S., Edwards, J. E., Gilbert, D., Rice, L. B., et al. (2009). Bad bugs, no drugs: no ESKAPE! An update from the Infectious diseases society of america. Clin. Infect. Dis. 48, 1-12. doi: 10.1086/595011

Chin, Y.-W., Balunas, M. J., Chai, H. B., and Kinghorn, A. D. (2006). Drug discovery from natural sources. AAPS J. 8, E239-E253. doi: 10.1007/ BF02854894

CLSI (2013). Performance Standards for Antimicrobial Susceptibility Testing; Twenty-Third Informational Supplement. Wayne, PA: Clinical \& Laboratory Standards Institute.

Cook, R. M., Lindsay, J. G., Wilkins, M. B., and Nimmo, H. G. (1995). Decarboxylation of malate in the Crassulacean acid metabolism plant Bryophyllum (Kalanchoe) fedtschenkoi (role of NAD-malic enzyme). Plant Phys. 109, 1301-1307. doi: 10.1104/pp.109.4.1301

Cruz, E., Reuter, S., Martin, H., Dehzad, N., Muzitano, M., Costa, S., et al. (2012). Kalanchoe pinnata inhibits mast cell activation and prevents allergic airway disease. Phytomedicine 19, 115-121. doi: 10.1016/j.phymed.2011. 06.030

Cumberbatch, A. (2011). An Ethnobotanical Survey of Medicinal Plant Usage in Salvador de Bahia, Brazil. Salvador: CGI Group.

Descoings, B. (2003). "Kalanchoe," in Illustrated Handbook of Succulent Plants: Crassulaceae, ed. U. Eggli (Berlin: Springer), 143-181.

Dittrich, P. (1976). Equilibration of label in malate during dark fixation of $\mathrm{CO} 2$ in Kalanchoë fedtschenkoi. Plant Phys. 58, 288-291. doi: 10.1104/pp.58.3.288

Gaind, K., and Gupta, R. (1971). Flavonoid glycosides from Kalanchoe pinnata. Planta Med. 20, 368-373. doi: 10.1055/s-0028-1099718

Gaind, K., and Gupta, R. (1973). Phenolic components from the leaves of Kalanchoe pinnata. Planta Med. 23, 149-153. doi: 10.1055/s-0028- 1099426

Herawati, M. H., and Husin, N. (2000). Berbagai jenis tumbuhan yang berkhasiat sebagai obat kecacingan. Media Litbang Kesehatan 10, 8-13.

Huang, H.-C., Lin, M.-K., Yang, H.-L., Hseu, Y.-C., Liaw, C.-C., Tseng, Y.-H., et al. (2013). Cardenolides and bufadienolide glycosides from Kalanchoe tubiflora and evaluation of cytotoxicity. Planta Med. 79, 1362-1369. doi: 10.1055/s-00331350646

Iqbal, S. M., Jamil, Q., Jamil, N., Kashif, M., Mustafa, R., and Jabeen, Q. (2016). Antioxidant, antibacterial and gut modulating activities of Kalanchoe laciniata. Acta Pol. Pharm. 73, 1221-1227.

Irenji, N., Pillai, S. K. G., and West-Jones, J. S. (2018). Serious life-threatening multifocal infection in a child, caused by panton-valentine leucocidinproducing Staphylococcus aureus (PVL-MSSA). BMJ Case Rep. 2018:bcr-2017222138. doi: 10.1136/bcr-2017-222138

Jazul, R. (1995). Antibacterial Property of the Malic Acid From the Leaves of Katakataka (Kalanchoe pinnata, Lam., Fam. Crassulaceae). Quezon: University of the Philippines.

Katsuura, Y., Cincere, B., Cason, G., and Osborn, J. (2018). Metastatic MSSA infection of the spine and extremities. BMJ Case Rep. 2018:bcr-2017-222778. doi: 10.1136/bcr-2017-222778

Kouitcheu Mabeku, L. B., Eyoum Bille, B., Tchouangueu, T. F., Nguepi, E., and Leundji, H. (2017). Treatment of Helicobacter pylori infected mice with Bryophyllum pinnatum, a medicinal plant with antioxidant and antimicrobial properties, reduces bacterial load. Pharm. Biol. 55, 603-610. doi: 10.1080/ 13880209.2016.1266668

Larasati, D., and Wahid, M. H. (2016). "In vitro anti-microbial efficacy of Kalanchoe pinnata leaves against Acinetobacter baumannii and methicillinresistant Staphylococcus aureus," in Proceedings of the Conference on 8th International Seminar of Indonesian Society for Microbiology, Jakarta.

Lebedeva, A., Zakharchenko, N., Trubnikova, E., Medvedeva, O., Kuznetsova, T., Masgutova, G., et al. (2017). Bactericide, immunomodulating, and wound healing properties of transgenic Kalanchoe pinnata synergize with antimicrobial peptide cecropin P1 in vivo. J. Immunol. Res. 2017:4645701. doi: 10.1155/2017/ 4645701

Maiti, S., Mukhopadhvay, R., and Bhattacharya, T. (1995). Chemical examination of Kalanchoe mortagei. Indian J. Pharmacol. 67, 113-114.

Majaz, Q. A., Nazim, S., Afsar, S., Siraj, S., and Siddik, P. M. (2011). Evaluation of antimicrobial activity of roots of Kalanchoe pinnata. Int. J. Pharm. Biol. Sci. 5:93.

Mendonça, F. S., Nascimento, N. C., Almeida, V. M., Braga, T. C., Ribeiro, D. P., Chaves, H. A., et al. (2018). An outbreak of poisoning by Kalanchoe blossfeldiana in cattle in northeastern Brazil. Trop. Anim. Health Prod. 50, 693-696. doi: 10.1007/s11250-017-1465-7

Milad, R., El-Ahmady, S., and Singab, A. N. (2014). Genus Kalanchoe (Crassulaceae): a review of its ethnomedicinal, botanical, chemical and pharmacological properties. Eur. J. Med. Plants 4, 86-104. doi: 10.9734/EJMP/ 2014/5901

Moniuszko-Szajwaj, B., Pecio, Ł., Kowalczyk, M., and Stochmal, A. (2016). New bufadienolides isolated from the roots of Kalanchoe daigremontiana (Crassulaceae). Molecules 21:243. doi: 10.3390/molecules21030243

Muzitano, M. F., Bergonzi, M. C., De Melo, G. O., Lage, C. L., Bilia, A. R., Vincieri, F. F., et al. (2011). Influence of cultivation conditions, season of collection and extraction method on the content of antileishmanial flavonoids from Kalanchoe pinnata. J. Ethnopharmacol. 133, 132-137. doi: 10.1016/j.jep.2010.09.020

Muzitano, M. F., Cruz, E. A., de Almeida, A. P., Da Silva, S. A., Kaiser, C. R., Guette, C., et al. (2006a). Quercitrin: an antileishmanial flavonoid glycoside from Kalanchoe pinnata. Planta Med. 72, 81-83.

Muzitano, M. F., Tinoco, L. W., Guette, C., Kaiser, C. R., Rossi-Bergmann, B., and Costa, S. S. (2006b). The antileishmanial activity assessment of unusual flavonoids from Kalanchoe pinnata. Phytochemistry 67, 2071-2077.

Muzitano, M. F., Falcão, C. A., Cruz, E. A., Bergonzi, M. C., Bilia, A. R., Vincieri, F. F., et al. (2009). Oral metabolism and efficacy of Kalanchoe pinnata flavonoids in a murine model of cutaneous leishmaniasis. Planta Med. 75, 307-311. doi: 10.1055/s-0028-1088382

Nielsen, A. H., Olsen, C. E., and Møller, B. L. (2005). Flavonoids in flowers of 16 Kalanchoe blossfeldiana varieties. Phytochemistry 66, 2829-2835. doi: 10.1016/j. phytochem.2005.09.041

Nimmo, G. A., Nimmo, H., Hamilton, I. D., Fewson, C. A., and Wilkins, M. B. (1986). Purification of the phosphorylated night form and dephosphorylated day form of phosphoenolpyruvate carboxylase from Bryophyllum fedtschenkoi. Biochem. J. 239:213. doi: 10.1042/bj2390213

Nwadinigwe, A. O. (2011). Antimicrobial activities of methanol and aqueous extracts of the stem of Bryophyllum pinnatum kurz (Crassulaceae). Afr. J. Biotechnol. 10, 16342-16346. doi: 10.5897/AJB11.1000

Obaseiki-Ebor, E. (1985). Preliminary report on the in vitro antibacterial activity of Bryophyllum pinnatum leaf juice. Afr. J. Med. Med. Sci. 14, 199-202.

Ofokansi, K., Esimone, C., and Anele, C. (2005). Evaluation of the in vitro combined antibacterial effect of the leaf extracts of Bryophyllum pinnatum (Fam: Crassulaceae) and Ocimum gratissimum (Fam: Labiatae). Plant Prod. Res. J. 9, 23-27.

Okwu, D. E., and Nnamdi, F. U. (2011). Two novel flavonoids from Bryophyllum pinnatum and their antimicrobial activity. J. Chem. Pharm. Res. 3, 1-10.

O’Neil, J. (2016). Tackling Drug-Resistant Infections Globally. Review on Antimicrobial Resistance. Available at: http://amr-review.org/sites/default/files/ 160518_Final\%20paper_with\%20cover.pdf

Pattewar, S. V. (2012). Kalanchoe pinnata: phytochemical and pharmacological profile. Int. J. Pharm. Sci. Res. 3:993. doi: 10.7439/ijpp.v2i1.223

Pattewar, S. V., Patil, D. N., and Dahikar, S. (2013). Antimicrobial potential of extract from leaves of Kalanchoe pinnata. Int. J. Pharm. Sci. Res. 4:4577.

Quave, C. L., Lyles, J. T., Kavanaugh, J. S., Nelson, K., Parlet, C. P., Crosby, H. A., et al. (2015). Castanea sativa (European Chestnut) leaf extracts rich in ursene and oleanene derivatives block Staphylococcus aureus virulence and pathogenesis without detectable resistance. PLoS One 10:e0136486. doi: 10. 1371/journal.pone.0136486

Quave, C. L., Plano, L. R., Pantuso, T., and Bennett, B. C. (2008). Effects of extracts from Italian medicinal plants on planktonic growth, biofilm formation and adherence of methicillin-resistant Staphylococcus aureus. J. Ethnopharmacol. 118, 418-428. doi: 10.1016/j.jep.2008.05.005

Quazi Majaz, A., Tatiya, A., Khurshid, M., Nazim, S., and Siraj, S. (2011). The miracle plant (Kalanchoe pinnata): a phytochemical and pharmacological review. Int. J. Res. Ayurveda Pharm. 2, 1478-1482. 
Rahman, M. M., Shiu, W. K. P., Gibbons, S., and Malkinson, J. P. (2018). Total synthesis of acylphloroglucinols and their antibacterial activities against clinical isolates of multi-drug resistant (MDR) and methicillin-resistant strains of Staphylococcus aureus. Eur. J. Med. Chem. 155, 255-262. doi: 10.1016/j.ejmech. 2018.05.038

Rajsekhar, P., Bharani, R., Ramachandran, M., Angel, K., and Rajsekhar, S. P. V. (2016). The "wonder plant" Kalanchoe pinnata (Linn.) pers.: a review. J. Appl. Pharm. Sci. 6, 151-158. doi: 10.7324/JAPS.2016.60326

Salam, A. M., and Quave, C. L. (2018). Opportunities for plant natural products in infection control. Curr. Opin. Microbiol. 45, 189-194. doi: 10.1016/j.mib.2018. 08.004

Sarkar, R., Mondal, C., Bera, R., Chakraborty, S., Barik, R., Roy, P., et al. (2015). Antimicrobial properties of Kalanchoe blossfeldiana: a focus on drug resistance with particular reference to quorum sensing-mediated bacterial biofilm formation. J. Pharm. Pharmacol. 67, 951-962. doi: 10.1111/jphp.12397

Sarker, S. D., Latif, Z., and Gray, A. I. (eds) (2005). Natural Products Isolation. New York, NY: Humana Press. doi: 10.1385/1592599559

Sarker, S.D., and Nahar, L. (2012). "An introduction to natural products isolation, in Natural Products Isolation, 3rd Edn, eds S. D. Sarker, and L. Nahar (New York,NY: Humana Press), 1-25.

SERNEC (2018). Southeast Regional Network of Expertise and Collections. Boone, NC: Appalachian State University.

Singleton, V. L., Orthofer, R., and Lamuela-Raventós, R. M. (1999). “Analysis of total phenols and other oxidation substrates and antioxidants by means of Folin-Ciocalteu reagent," in Methods in Enzymology, eds J. Abelson, M. Simon, G. Verdine, and A. Pyle (Cambridge, MA: Academic Press), 152-178.

Supratman, U., Fujita, T., Akiyama, K., and Hayashi, H. (2000). New insecticidal bufadienolide, bryophyllin C, from Kalanchoe pinnata. Biosci. Biotechnol. Biochem. 64, 1310-1312. doi: 10.1271/bbb.64.1310

Tatsimo, S. J. N., de Dieu Tamokou, J., Havyarimana, L., Csupor, D., Forgo, P., Hohmann, J., et al. (2012). Antimicrobial and antioxidant activity of kaempferol rhamnoside derivatives from Bryophyllum pinnatum. BMC Res. Notes 5:158. doi: 10.1186/1756-0500-5-158

Torres-Santos, E., Da Silva, S., Costa, S., Santos, A., Almeida, A., and RossiBergmann, B. (2003). Toxicological analysis and effectiveness of oral Kalanchoe pinnata on a human case of cutaneous leishmaniasis. Phytother. Res. 17, 801803. doi: $10.1002 /$ ptr. 1242

Ürményi, F. G. G., Saraiva, G. D. N., Casanova, L. M., Matos, A. D. S., Magalhães Camargo, L. M., Romanos, M. T. V., et al. (2016). Anti-HSV-1 and HSV2 flavonoids and a new kaempferol triglycoside from the medicinal plant Kalanchoe daigremontiana. Chem. Biodivers. 13, 1707-1714. doi: 10.1002/cbdv. 201600127

USDA/NRCS (2013). The PLANTS Database. Greensboro, North Carolina: National Plant Data Team. Available at: http://plants.usda.gov [accessed September 7, 2018].

van der Meer, J. W., Fears, R., Davies, S. C., and ter Meulen, V. (2014). Antimicrobial innovation: combining commitment, creativity and coherence. Nat. Rev. Drug Discov. 13, 709-710. doi: 10.1038/nrd4448

Vera-Marín, B., and Sánchez-Sáen, M. (2016). Plantas medicinales y predictibilidad de uso en algunas veredas del corregimiento de San Cristóbal (Antioquia), Colombia. Actualidades Biológicas 38, 167-180.

WHO (2017). Global Antimicrobial Resistance Surveillance System (GLASS) Report: Early Implementation 2016-2017. Geneva: World Health Organization.

Wright, G. D. (2017). Opportunities for natural products in 21 st century antibiotic discovery. Nat. Prod. Rep. 34, 694-701. doi: 10.1039/c7np00019g

Zakharchenko, N., Lebedeva, A., Furs, O., Rukavtsova, E., Schevchuk, T., Rodionov, I., et al. (2016). Producing marker-free Kalanchoe plants expressing antimicrobial peptide cecropin P1 gene. Russ. J. Plant Physiol. 63, 273-282. doi: $10.1134 /$ S1021443716020163

Conflict of Interest Statement: The authors declare that the research was conducted in the absence of any commercial or financial relationships that could be construed as a potential conflict of interest.

Copyright (c) 2019 Richwagen, Lyles, Dale and Quave. This is an open-access article distributed under the terms of the Creative Commons Attribution License (CC BY). The use, distribution or reproduction in other forums is permitted, provided the original author(s) and the copyright owner(s) are credited and that the original publication in this journal is cited, in accordance with accepted academic practice. No use, distribution or reproduction is permitted which does not comply with these terms. 